\title{
Causes, Clinical Features, and Outcomes From a Prospective Study of Drug-Induced Liver Injury in the United States
}

\author{
Naga Chalasani ${ }^{*}, \ddagger$, Robert J. Fontana $\S$, Herbert L. Bonkovsky $\|, \uparrow, \#$, Paul B. Watkins $\llbracket$, \\ Timothy Davern $^{\star *}$, Jose Serrano $\neq \ddagger$, Hongqiu Yang ${ }^{\S}$, James Rochon $\S$, and for the Drug \\ Induced Liver Injury Network (DILIN)
}

*Department of Medicine, Indiana University School of Medicine, Indianapolis, Indiana ${ }^{*}$ Clarian/ Indiana University Digestive Diseases Center, Indianapolis, Indiana §Department of Internal Medicine, University of Michigan Medical School, Ann Arbor, Michigan "Cannon Research Center and Center for Liver and Digestive Diseases, Carolinas Medical Center, Charlotte, North Carolina "Department of Internal Medicine, University of North Carolina at Chapel Hill, Chapel Hill, North Carolina \#Department of Internal Medicine, University of Connecticut, Farmington, Connecticut ${ }^{* *}$ Department of Internal Medicine, University of California, San Francisco, San Francisco,

\section{(C) 2008 by the AGA Institute}

Address requests for reprints to: Naga Chalasani, MD, Indiana University School of Medicine, RG 4100, 1050 Wishard Boulevard, Indianapolis, Indiana 46202. nchalasa@iupui.edu; fax: (317) 278-1949.

Supplementary Data

Note: To access the supplementary material accompanying this article, visit the online version of Gastroenterology at www.gastrojournal.org, and at doi: 10.1053/j.gastro.2008.09.011.

R.J.F. has served on the speakers' bureau or as a paid consultant during the past 12 months to Roche, Bristol-Myers Squibb, Vertex Pharmaceuticals, and Gilead Sciences.

H.L.B. served as a paid advisor to InfaCare Pharmaceuticals, Novartis Pharmaceuticals, and Ovation Pharmaceuticals. He is on the speakers' bureau of Ovation Pharmaceuticals. He receives support for research studies from the American Porphyria Foundation, Merck, Novartis, Roche, and Vertex. During the past 12 months, he has served as an expert witness for plaintiffs in litigation regarding suspected drug-induced liver injury.

P.B.W. served as a paid consultant in the preceding 12 months to the following pharmaceutical companies: Actelion, Aldus, Astellas, Bristol-Myers Squibb, Boehringer-Ingleheim, Danube, Endo, FibroGen, Glaxo-SmithKline, Hoffmann-La Roche, Imtech, King, Millennium, Merck, Novartis, Nuon, Orion, Pfizer, Pharmasset, Schering Plough, TAP, Valiant, VIA, and Wyeth. He served as both a plaintiff and a defense expert in litigation involving suspected drug-induced liver injury.

T.D. served as a paid consultant to Entelos and FibroGen. He served as both a plaintiff and a defense expert in litigation involving suspected drug-induced liver injury.

J.S., H.Y., and J.R. have no potential conflicts of interest to report.

Members of DILIN include the following: Clinical Centers-Indiana University: Naga Chalasani, MD (primary investigator), Raj Vuppalanchi, MD (coinvestigator), Jean Molleston, MD (coinvestigator), Lawrence Lumeng, MD (coinvestigator), Audrey Corne (research coordinator), Angie Plummer (research coordinator); University of Connecticut: Herbert Bonkovsky, MD (primary investigator), Petr Protiva, MD (coinvestigator), James Freston, MD, PhD (coinvestigator), Robert Rosson, MD (coinvestigator), Robert A. Levine, MD (satellite site investigator), Benedict Maliakkal, MD (satellite site investigator), Paul Appleton, MD (research coordinator), Mariola Smialek, RN (research coordinator); University of Michigan: Robert J. Fontana, MD (primary investigator), Hari Conjeevaram, MD (coinvestigator), Stuart Gordon, MD (satellite site investigator), Suzanne Welch (research coordinator), Jessica Worley (research coordinator), Jordan Kridler (research coordinator); University of North Carolina: Paul Watkins, MD (primary investigator), Paul Hayashi, MD (coinvestigator), Mark Russo, MD (coinvestigator), the late Harry Guess, MD, PhD (coinvestigator), Kimberly Beaver, MD (satellite site investigator), Alastair Smith, MD (satellite site investigator), James Lewis, MD (satellite site investigator), Susan Pusek (research coordinator); University of California, San Francisco: Tim Davern, MD (primary investigator), Maurizo Bonacini, MD (coinvestigator), Kristine Partovi (research coordinator). Data Coordinating Center- Duke Clinical Research Institute: James Rochon, MD (primary investigator), John McHutchison, MD (coinvestigator), Don Rockey, MD (coinvestigator), Mary Maggio (project manager), Hongqiu Yang, PhD (biostatistician); National Institute of Diabetes and Digestive and Kidney Diseases Scientists: Jose Serrano, MD (project officer), Leonard Seeff, MD, Jay Hoofnagle, MD, Mark Avigan, MD, and John Senior, MD, employees of the US Food and Drug Administration, have participated in selected aspects of DILIN activities. Members of DILIN thank all coinvestigators, research coordinators, referring physicians, and most importantly patients for their participation in this study, as well as the late Harry Guess, MD, PhD (Professor of Epidemiology and Pediatrics, University of North Carolina at Chapel Hill) for his contributions to DILIN. 
California ¥‡Liver Disease Research Branch, National Institute of Diabetes and Digestive and Kidney Diseases, National Institutes of Health, Bethesda, Maryland $\$ \S$ Duke Clinical Research Institute, Durham, North Carolina

\section{Abstract}

Background \& Aims-Idiosyncratic drug-induced liver injury (DILI) is among the most common causes of acute liver failure in the United States, accounting for approximately $13 \%$ of cases. A prospective study was begun in 2003 to recruit patients with suspected DILI and create a repository of biological samples for analysis. This report summarizes the causes, clinical features, and outcomes from the first 300 patients enrolled.

Methods-Patients with suspected DILI were enrolled based on predefined criteria and followed up for at least 6 months. Patients with acetaminophen liver injury were excluded.

Results-DILI was caused by a single prescription medication in $73 \%$ of the cases, by dietary supplements in $9 \%$, and by multiple agents in $18 \%$. More than 100 different agents were associated with DILI; antimicrobials $(45.5 \%)$ and central nervous system agents $(15 \%)$ were the most common. Causality was considered to be definite in $32 \%$, highly likely in $41 \%$, probable in $14 \%$, possible in $10 \%$, and unlikely in $3 \%$. Acute hepatitis $\mathrm{C}$ virus (HCV) infection was the final diagnosis in 4 of 9 unlikely cases. Six months after enrollment, $14 \%$ of patients had persistent laboratory abnormalities and $8 \%$ had died; the cause of death was liver related in $44 \%$

Conclusions-DILI is caused by a wide array of medications, herbal supplements, and dietary supplements. Antibiotics are the single largest class of agents that cause DILI. Acute HCV infection should be excluded in patients with suspected DILI by HCV RNA testing. The overall 6month mortality was $8 \%$, but the majority of deaths were not liver related.

Drug-induced liver injury (DILI) is a serious health problem that impacts patients, physicians, the pharmaceutical industry, and government regulators. ${ }^{1-3}$ DILI is the most common cause of death from acute liver failure and accounts for approximately $13 \%$ of cases of acute liver failure in the United States. ${ }^{4}$ DILI is the most frequent adverse drug event leading to abandonment of otherwise promising new drug candidates during preclinical or clinical development, failure of drugs to achieve approval by the regulatory agencies, and withdrawal or restriction of prescription drug use after initial approval. ${ }^{1-3}$

Idiosyncratic DILI from any single medication is a rare clinical event occurring in less than 1 per 10,000 to 100,000 of subjects who take the drug. The risk factors for this rare occurrence and the pathogenesis are poorly understood. ${ }^{1-3,5,6}$ Most cases of DILI are unpredictable and generally believed to be due to an immunoallergic reaction or an abnormality in the metabolism of the agent and lack a dose relationship, although a dose threshold has been suggested recently. ${ }^{5,7,8}$ The clinical presentation of DILI covers a wide spectrum, from asymptomatic liver test abnormalities to symptomatic acute liver disease, prolonged jaundice and disability, or overt acute or subacute liver failure. The recognition and diagnosis of DILI are often difficult and delayed due to the need to exclude more common competing causes of liver injury.

There is a growing awareness that nonprescription drugs, including herbal remedies and other over-the-counter dietary supplements, are also important causes of DILI. ${ }^{9,10}$ For example, organ toxicity associated with formulations that contain ephedra as a major ingredient has led to their removal by regulatory authorities in some Western European countries, ${ }^{11,12}$ and recent reports from the United States have emphasized the risks of use of extracts of Chinese green tea (Camellia sinensis) that contain catechins as a major 
ingredient. ${ }^{13-15}$ Because numerous dietary supplements are consumed by large numbers of US adults on a regular basis, ${ }^{16}$ the hepatotoxicity of dietary supplements may be significantly underestimated.

It has been hypothesized that host genetic, immunologic, and environmental factors are important in the pathogenesis of DILI. ${ }^{1-3,5,6,17}$ Thus, there is a growing expectation that use of modern genome-wide association studies and other genetic analyses, coupled with careful phenotyping of subjects, will improve the ability to identify subjects at high or low risk for developing DILI from various drugs. This is an important part of the promise of personalized medicine. ${ }^{18,19}$

The Drug-Induced Liver Injury Network (DILIN) was established in 2003 as a cooperative agreement among the National Institutes of Health, 5 academic clinical centers, and a data coordinating center. ${ }^{20} \mathrm{~A}$ major emphasis of DILIN has been to establish a protocol for the identification and enrollment of patients with clinically significant DILI into a prospective observational study and to create a registry and specimen repository of biological samples that could be used for mechanistic studies on the etiology and prevention of DILI. The design and development of the DILIN prospective study protocol ${ }^{21}$ and the process of causality assessment ${ }^{22}$ have been presented elsewhere. In this report, we describe the implicated agents, presenting clinical and laboratory features, and short-term outcomes of the first 300 subjects enrolled in the ongoing DILIN prospective study.

\section{Patients and Methods}

The DILIN prospective study is an ongoing multicenter observational study. The study design and procedures were approved by the institutional review board of each clinical center site, and all enrolled patients provided written, fully informed consent. The study design has been described in detail elsewhere. ${ }^{21}$ In brief, patients ( 2 years of age or older) were enrolled in this study if there was a strong clinical suspicion that a liver injury event was caused by a medication or an herbal agent occurring within 6 months before enrollment. Additionally, patients must meet one of the following biochemical criteria for enrollment into this study: (1) aspartate aminotransferase (AST) or alanine aminotransferase (ALT) level $>5$ times the upper limit of normal (ULN) or alkaline phosphatase level $>2$ times the ULN (or pretreatment baseline if baseline level is abnormal) on 2 consecutive occasions, (2) total serum bilirubin level $>2.5 \mathrm{mg} / \mathrm{dL}$ along with elevated AST or ALT or alkaline phosphatase level, or (3) international normalized ratio (INR) $>1.5$ with elevated AST or ALT or alkaline phosphatase level. Known or suspected acetaminophen toxicity and a history of bone marrow or liver transplantation before the liver injury event were exclusion criteria. Patients with underlying hepatitis $\mathrm{C}$ virus (HCV), hepatitis B virus, or nonalcoholic fatty liver disease were eligible if they developed superimposed DILI; however, those with other types of underlying chronic liver disease (eg, autoimmune liver disease, sclerosing cholangitis) were ineligible. Subjects with known or suspected acetaminophen hepatotoxicity or a history of bone marrow or liver transplantation were excluded.

Eligible patients were seen for a baseline study visit, during which a medical history and a detailed history of the liver injury event and exposure to the implicated agent(s) were obtained and clinical, laboratory, histologic, and imaging results were extracted from the medical chart. At this time, further laboratory testing was performed to more fully characterize the DILI event and to exclude competing etiologies. ${ }^{1,2}$ All enrolled individuals were followed up for at least 6 months, and those with evidence of chronic DILI were asked to return at 12 and 24 months. Chronic DILI was defined as persistent liver-related laboratory, radiologic, or histologic abnormalities at 6 months after DILI recognition. ${ }^{21}$ 
In the clinical characterization of DILI, the ratio of serum ALT (as a multiple of its ULN) to serum alkaline phosphatase (as a multiple of its ULN) has been designated as the R (for ratio) value. Hepatocellular DILI is defined as $R \geq 5$, cholestatic as $R \leq 2$, and "mixed" as $R$ $\geq 2$ to $\mathrm{R}<5.1,2$ For the purpose of this report, "DILI recognition" was defined as the time point when patients met the enrollment criteria. For brevity, herbal remedies, natural products, vitamins, minerals, and other dietary supplements are referred to hereafter as "dietary supplements."

The diagnosis of DILI and the causal relationship between the liver injury event and the implicated agent(s) were evaluated in a formal and standardized fashion by the DILIN Causality Committee. ${ }^{22}$ The causality assessment was conducted for each case by using 2 different causality instruments: the widely used Roussel Uclaf Causality Assessment Method (RUCAM) ${ }^{23-25}$ and assigning a DILIN causality score based on the consensus of at least 3 hepatologist members of the committee. ${ }^{22}$ The RUCAM provides a semiquantitative evaluation of causality by assigning -3 to +3 points to each of its 6 domains. Based on the final score, a causal relationship between the implicated agent and the liver injury event was categorized as highly probable (>8), probable (6-8), possible (3-5), unlikely (1 or 2), or excluded $(<0) .{ }^{23-25}$

The DILIN causality score categorizes the strength of causal association between the implicated agent and the liver injury event as definite (>95\% likelihood), highly likely $(75 \%-95 \%)$, probable $(50 \%-74 \%)$, possible $(25 \%-49 \%)$, and unlikely $(<25 \%) \cdot{ }^{21}$ In addition, the severity of each DILI episode was categorized as one of 5 levels (mild, moderate, moderate-hospitalized, severe, and fatal/transplant) as described elsewhere. ${ }^{21}$

\section{Data Management and Statistical Analyses}

Demographic and clinical data for the first 300 patients enrolled into this ongoing study were extracted on December 1, 2007. Because causality adjudication lags behind data collection, causality assessment was completed on only 254 of the 300 cases. No specific sample size calculations were made to choose a sample size of 300 patients for this largely descriptive report. Simple descriptive statistics, that is, mean \pm SD, median with 25 th and 75th percentiles, and frequency distributions, were used to characterize the cohort. Betweengroup differences were assessed using the Kruskal-Wallis test for continuous variables and the likelihood ratio test for categorical variables. For identifying predictors of DILI severity, the severe and fatal/transplant categories were combined into 1 group (severe DILI) and compared with the other 3 groups combined (mild/moderate DILI) using the likelihood ratio test. Subsequently, multivariable logistic regression analysis, consisting of selected clinically relevant variables (age, sex, race) and those with $P<.1$ on univariate analysis (alcohol consumption, diabetes, duration between exposure and DILI recognition, and pattern of liver injury), was conducted to identify factors independently associated with advanced DILI. All statistical analyses were performed using SAS version 8.1 (SAS Institute, Inc, Cary, NC). $P<.05$ was considered statistically significant.

\section{Results}

\section{Presenting Features}

The 300 patients included in this report were enrolled between September 2004 and December 2007. Selected demographic and clinical characteristics are shown in Table 1. Ninety-three percent were adults ( $\geq 18$ years), $18 \%$ were older than 65 years, and $60 \%$ were women. Six percent of patients had known liver disease before the onset of DILI, and 3\% had underlying human immunodeficiency virus infection. Sixty-nine percent had jaundice during the DILI episode, and 60\% were hospitalized. The median duration between first 
exposure to the implicated agent and DILI recognition was 42 (20-117) days. The duration of exposure before DILI recognition was not different depending on gender or race. The median duration between DILI recognition and study enrollment was 49.5 (21-104) days. At the time of DILI recognition, the values for serum biochemistries (mean \pm SD) were as follows: ALT, $788 \pm 967 \mathrm{U} / \mathrm{L}$; alkaline phosphatase, $295 \pm 272 \mathrm{U} / \mathrm{L}$; total bilirubin, $6.3 \pm$ $6.3 \mathrm{mg} / \mathrm{dL}$; and INR, $1.5 \pm 0.9$.

\section{Causative Agents}

A single prescription medication was implicated in $217(73 \%)$ of the 300 subjects, whereas single or multiple dietary supplements were implicated in 28 patients (9\%). In 55 patients (18\%), more than one prescription medicine or a combination of prescription medicine and dietary supplement(s) was implicated. A complete list of agents implicated is provided in Supplementary Table 1 (see supplemental material online at www.gastrojournal.org). Among subjects in whom a single suspect prescription medication was implicated, the major classes of agents were as follows: antimicrobials (antibacterial agents, antiviral agents, antituberculosis agents, and so on) in $45.5 \%$, central nervous system agents (antiepileptic agents, antidepressants, antipsychotics) in 15\%, immunomodulatory agents in 5.5\%, analgesics (nonsteroidal agents, muscle relaxants) in 5\%, antineoplastic agents in 4\%, antihypertensive agents in 5\%, and lipid-lowering agents in 3.4\%. The most common single implicated agents were amoxicillin/clavulanate $(n=23)$, nitrofurantoin $(n=13)$, isoniazid $(n$ $=13)$, and trimethoprim-sulfamethoxazole $(n=13)$.

\section{Values of R: Types of DILI}

The R value was calculated in 298 patients who had both serum ALT and alkaline phosphatase values available on the day of DILI recognition. A total of $169(57 \%)$ were classified as hepatocellular, $68(23 \%)$ as cholestatic, and $61(20 \%)$ as mixed. The clinical and laboratory features of patients with the 3 patterns of DILI are shown in Table 1. Noteworthy were the younger age and higher proportion of women with hepatocellular injury in comparison with cholestatic and mixed liver injury. The absence of preexisting chronic liver disease in the mixed DILI is noteworthy but of unclear significance.

\section{Causality Assessment}

Causality adjudication has been completed in 254 of the 300 patients. Using expert opinion, the likelihood of DILI as the reason for the liver injury was deemed definite in $32 \%$, highly likely in $41 \%$, probable in $14 \%$, and possible in $10 \%$. In 9 individuals, DILI was deemed unlikely to be responsible for the liver injury event; in these cases, the final diagnoses were acute hepatitis $\mathrm{C}$ in 4, unknown in 3, and polymyositis and benign recurrent intrahepatic cholestasis in 1 each (Supplementary Table 2; see supplemental material online at www.gastrojournal.org).

The RUCAM scores assigned by the site investigator were available for 192 DILI cases caused by a single prescription agent and were ranked as highly probable in $10 \%$, probable in $45 \%$, possible in $36 \%$, unlikely in $5 \%$, and excluded in $4 \%$. Because the RUCAM is designed to attribute causality to an individual implicated agent rather than to assess the global likelihood of DILI, cases with multiple possible causative agents have more than one RUCAM score and those data are reported elsewhere. ${ }^{22}$

\section{Course of Liver Injury}

Following clinical presentation, the peak serum biochemistries (mean \pm SD) were as follows: ALT, $985 \pm 1168 \mathrm{U} / \mathrm{L}$; alkaline phosphatase, $390 \pm 382 \mathrm{U} / \mathrm{L}$; total bilirubin, $11.4 \pm$ $10.2 \mathrm{mg} / \mathrm{dL}$; and INR, $1.6 \pm 1.4$. The degree of severity of the liver disease was judged to be 
mild in $27 \%$, moderate in $19 \%$, moderate-hospitalized in $33 \%$, severe in $15 \%$, and resulting in death or liver transplantation in 6\%. Selected demographic and clinical characteristics of patients with severe (severe and fatal/transplant cases combined) and mild/moderate (all other cases) disease and the results of the univariate analyses are shown in Table 2 . In the multivariate logistic analysis including age, sex, race, coexistent diabetes mellitus, alcohol consumption, smoking, biochemical pattern of liver injury, and the duration between first exposure and DILI recognition as covariates, only the presence of diabetes and alcohol consumption were independently associated with severe DILI (Supplementary Table 3; see supplemental material online at www.gastrojournal.org). The presence of diabetes mellitus was an independent risk factor for severe DILI (odds ratio, 2.69, 95\% confidence interval, 1.14-6.45), whereas any alcohol use in the preceding 12 months was a negative predictor of severe DILI (odds ratio, 0.33 ; 95\% confidence interval, 0.15- 0.76). Interestingly, the median duration between first exposure to the implicated agent and DILI recognition was significantly longer in severe cases than in mild/ moderate cases (65.5 [33-263] vs 35.5 [1989] days; $P=.006$ ), but this association was not statistically significant in the multivariate analysis (Table 2).

The median duration between DILI recognition and the peak value for ALT was $1(0-7)$ days, for alkaline phosphatase was $4(0-16)$ days, and for total bilirubin was $7(0-17)$ days. There was no statistically significant relationship between any of these durations and age, pattern of liver injury, implicated agent categories (single prescription agent vs dietary supplement[s] vs multiple agents), and causality or severity scores.

At the 6-month follow-up visit, $13.6 \%$ of enrolled patients had met predefined criteria for chronic DILI, $8 \%$ had died, and $2.1 \%$ had undergone liver transplantation. ${ }^{21}$ Features of the implicated agent, patient age, and pattern of DILI were not associated with chronicity, mortality, or need for transplantation (Table 3). Among DILI subjects with jaundice at or after presentation (total serum bilirubin level $\geq 2.5 \mathrm{mg} / \mathrm{dL}$ ), the median duration between peak bilirubin level and 50\% reduction in total bilirubin level was 13 days (4-30 days) and the median duration from peak bilirubin level to a level $<2.5 \mathrm{mg} / \mathrm{dL}$ was 26.5 days (3-54 days) (Table 4). The patterns and times of changes in serum bilirubin levels did not correlate with clinical features of the DILI cases.

Table 5 shows selected characteristics and causes of death in the 27 patients with suspected DILI who died or received liver transplantation within 180 days following the DILI event. Interestingly, among the 18 patients who died, the cause of death was judged by the investigators to be liver related in only $8(44 \%)$. The mortality in patients with hepatocellular DILI with peak serum total bilirubin level $\geq 2.5 \mathrm{mg} / \mathrm{dL}$ was significantly higher than in those with hepatocellular DILI with serum total bilirubin level $<2.5 \mathrm{mg} / \mathrm{dL}$ ( $13.4 \%$ vs $2.4 \%$, respectively; $P=.04$ ). However, the mortality rate in patients with cholestatic DILI with serum total bilirubin level $\geq 2.5 \mathrm{mg} / \mathrm{dL}$ was not statistically different than in those with cholestatic DILI with bilirubin level $<2.5 \mathrm{mg} / \mathrm{dL}$ ( $15 \%$ vs $10 \%$, respectively; $P=.66$ ). Among 17 patients who died from liver failure or received a transplant, 14 had hepatocellular DILI, whereas 2 had mixed and 1 had cholestatic DILI (Table 5).

\section{Dietary Supplements}

Dietary supplements were implicated in 33 subjects with DILI; one or more dietary supplements were implicated in 28 , whereas they were implicated in combination with one or more prescription agents in the remaining 5. In many cases, multiple agents were being used; even when a single preparation was used, it often contained multiple herbal or nutritional components, so that attribution of liver injury to a single component was rarely possible. The names of all implicated herbal agent(s) are shown in Supplementary Table 1 
(see supplemental material online at www.gastrojournal.org). The stated reasons for consuming these dietary supplements were muscle building $(n=11)$, weight loss $(n=8)$, insomnia $(n=2)$, general well-being $(n=2)$, preventing common cold $(n=2)$, increasing energy levels $(\mathrm{n}=2)$, and hot flashes $(\mathrm{n}=1)$. Compared with 217 cases due to single prescription medications, 28 DILI cases caused by dietary supplements showed few differences, none of which were statistically significant (Supplementary Table 4; see supplemental material online at www.gastrojournal.org). Patients with DILI due to dietary supplements had higher mean levels of serum total bilirubin ( $14.7 \pm 13$ vs $10.6 \pm 9.9 \mathrm{mg} / \mathrm{dL}$; $P=.11$ ) and longer median duration for the jaundice to resolve (68 vs 35 days; $P=.08$ ), but these trends were of borderline statistical significance. There were no instances of StevensJohnson syndrome or death due to dietary supplements, but one subject with suspected DILI due to CVS Spectravite developed acute liver failure necessitating liver transplantation.

\section{Discussion}

This is an initial analysis of an ongoing prospective study of DILI being performed in the United States, the primary aim of which is to develop well-characterized cases of medication-related liver injury on which to conduct hypothesis-driven research aimed at developing means to diagnose, prevent, and treat DILI. Among the first 300 cases identified, more than 100 different medications, herbal supplements, and dietary supplements were implicated. Newly identified hepatotoxic drugs that had received Food and Drug Administration warnings between 2003 and 2007 were identified by DILIN (eg, telithromycin, leflunomide, duloxetine), suggesting that a prospective network such as DILIN may be able to provide early detection of the hepatotoxic potential of newly released medications. An important finding was that more than one agent was implicated in causing liver injury in $\sim 20 \%$ of cases, a frequency significantly higher than the $9 \%$ reported from a similar study that had been conducted in Spain. ${ }^{26}$ The reason for this difference is unclear, but it may reflect higher use of medicines by the US population. Consistent with previous reports, antimicrobials represented the single largest class of agents to have caused DILI. ${ }^{26-29}$ It remains unclear why antimicrobials have such a high propensity to cause DILI, but it may be related to greater use of antimicrobials in the general population or biological reasons such as underlying infection and inflammation conferring increased susceptibility. ${ }^{30}$

The proportion of cases with suspected DILI caused by dietary supplements was nearly $10 \%$ and is higher than reported in the Spanish registry, likely reflecting a greater use of these products by the US population. ${ }^{26}$ None of the subjects with suspected DILI caused by dietary supplements were children. The intake of these compounds may be infrequent in children; however, we cannot exclude the possibility that children may be less susceptible than adults to hepatotoxicity by dietary supplements. Compounds that claim to promote muscle building and weight loss were the 2 most common classes of dietary supplements, accounting for nearly $60 \%$ of cases. The total number of cases of DILI due to dietary and herbal supplements was small $(\mathrm{n}=28)$, and no single supplement was responsible for more than one case, although, as a class, supplements that include extracts of green tea as a major ingredient caused at least 6 of the 28 cases.

The findings regarding the rate of change of serum total bilirubin levels after onset of DILI may be of practical relevance in monitoring and counseling patients with DILI (Tables 3 and 4). The total serum bilirubin level reached its peak an average of 1 week after DILI recognition, and this timing was independent of patient age, pattern of liver injury, or whether caused by prescription agents or dietary supplements. Among patients with jaundice, it took nearly 1 month on average for the jaundice to resolve, but this interval was longer in the elderly, in patients with cholestatic forms of DILI, or in patients with DILI caused by dietary supplements. 
Acute hepatitis $\mathrm{C}$ was the final diagnosis in 4 of the 9 cases that were adjudicated as "unlikely" to be DILI. Anti-HCV was initially negative in 2 subjects, but they subsequently seroconverted to detectable viremia. In 2 other patients, initial testing revealed the presence of anti-HCV, but the site investigators at the time of enrollment believed them to represent chronic rather than acute hepatitis $\mathrm{C}$, largely because both patients lacked recent risk factors for acquiring viral hepatitis. However, the availability of additional clinical data (eg, liver histology) made HCV infection rather than DILI the likely explanation for the acute event (Supplementary Table 2). These 4 patients did not admit to high-risk behaviors (eg, recent drug abuse), but 3 had been hospitalized recently. These findings suggest that, even in the absence of risk factors, acute hepatitis $\mathrm{C}$ should be excluded by testing for serum HCV RNA in all patients with suspected DILI, especially if there is a history of recent hospitalization and a hepatocellular pattern of injury. In fact, in light of these findings, our protocol was amended to obtain HCV RNA at the baseline visit in all enrolled patients. As better diagnostic tests become available for specific causes of acute liver injury, more cases of suspected DILI may be found to have other etiologies. Indeed, in a recent series from the United Kingdom, retrospective testing for antibody to hepatitis E virus in 69 patients with presumed DILI identified 6 patients with probably acute hepatitis E. ${ }^{31}$

In a cohort study of 461 patients with DILI from Spain, female sex, hepatocellular patterns of liver injury, and total serum bilirubin level on presentation were identified as independent predictors of acute liver failure. ${ }^{26}$ In the current US study, there was no relationship between female sex and severity of DILI; furthermore, the positive association between hepatocellular injury and severity of DILI was of borderline significance. Total serum bilirubin levels were higher in patients with severe DILI, but bilirubin is used in the criteria to define severity and therefore was not used in the multivariate analysis of factors correlating with severity and outcome. In this study, the presence of diabetes mellitus was found to be an independent risk factor for severe DILI. Diabetes was not considered as a covariate in previous studies from Spain ${ }^{26}$ and Sweden. ${ }^{29}$ However, epidemiologic and animal studies suggest that diabetes is associated with an increased incidence of acute liver failure and severity of DILI. ${ }^{32-35}$ Alcoholism is generally believed to be a risk factor for developing DILI (particularly injury due to acetaminophen), and, in the RUCAM causality instrument, points are given in favor of DILI for alcohol intake. However, outside of acetaminophen and isoniazid toxicity, the association between alcohol consumption and susceptibility to DILI has not been evaluated rigorously. ${ }^{1-3}$ The finding that alcohol consumption protected against severe DILI in this study was surprising and of uncertain significance. A major difficulty is the variability of definitions of alcohol intake and alcoholism in various studies and causality instruments. In this study, alcohol consumption was defined as any alcohol intake in the preceding 12 months. Additional studies are needed to determine if alcohol consumption may play a role in DILI susceptibility by comparing subjects with DILI with suitably matched controls without DILI. Previous studies also have shown associations between severe DILI and older age ${ }^{329}$ and eosinophil counts, ${ }^{35}$ but these were not confirmed in this study. The latter may not have been identified due to the lag between DILI recognition and study enrollment (median $\sim 42$ days). In a study of 95 patients with suspected DILI from Japan, ${ }^{36}$ the duration of exposure to the implicated agent was longer in cases of acute liver failure compared with less severe cases $(81 \pm 89$ vs $30 \pm 44$ days, respectively; $P<.0001$ by univariate analysis). In this study, the duration of exposure to the implicated agent was significantly longer in individuals with severe DILI, but this relationship was not statistically significant on multivariate analysis.

The mortality rate of DILI is generally high, particularly in cases with jaundice and a hepatocellular pattern of injury, colloquially known as "Hy's rule." 37 The $8 \%$ mortality rate in this study is in general agreement with prior reports. ${ }^{26,29}$ Although patients with cholestatic DILI have been thought to have a better prognosis than those with hepatocellular 
DILI, ${ }^{37}$ this association was not found in this study and has not been consistently found in recent large case series from Europe ${ }^{26,29}$ (Table 6). Importantly, most of the fatalities in patients with cholestatic forms of DILI were due to reasons other than liver failure (Table 5). The mortality rate of hepatocellular forms of liver injury with jaundice in this study was $15 \%$, which is compatible with Hy's rule, which states that the average mortality rate in patients with jaundice and a hepatocellular pattern of injury from DILI is at least $10 \%{ }^{38}$

Limitations of our study include the potential for selection bias, the arbitrary laboratory entry criteria, and the lack of international standards for diagnosing DILI. However, all patients were prospectively studied and had undergone a complete serologic, radiologic, and clinical assessment by experienced hepatologists. In addition, causality was determined by a committee of experts using standardized terminology. ${ }^{21}$ Overall, we may have enrolled patients with more severe DILI than is encountered in the general population and in other prior studies. ${ }^{39}$ This may be due to the fact that hospitalized patients were more likely to be referred to a DILIN investigator or undergo a complete evaluation. In addition, nearly $50 \%$ of our patients had undergone liver biopsy. However, a reasonable number of pediatric patients were enrolled (7\%) and a broad distribution of race/ethnicities was enrolled. In addition, our limited sample size precludes robust analysis of risk factors for DILI outcome. We plan to conduct additional multivariate analyses once the total enrollment increases. Finally, susceptibility risk factors for DILI will require comparing enrolled patients with control patients who took the same medication but did not develop liver injury. Appropriate controls will be recruited as the number of DILI cases caused by individual medications increases in number.

In summary, DILI in the United States is caused by a wide variety of prescription and nonprescription medications, nutritional supplements, and herbal supplements. The antimicrobials represent the single largest class of agents that cause DILI, accounting for at least $45 \%$ of cases. At least $20 \%$ of patients with DILI ingest more than one potentially hepatotoxic agent. Acute hepatitis $\mathrm{C}$ should be carefully excluded before attributing a case of acute liver injury to DILI. In this study, coexistent diabetes mellitus was an independent risk factor for more severe DILI, while alcohol consumption was a negative predictive factor. DILI with jaundice from hepatocellular liver injury carries a high mortality rate, but the mortality rate associated with cholestatic forms of DILI is also appreciable although often caused by worsening of the underlying condition or an unrelated disease. DILI still represents an important and problematic cause of acute liver disease in the United States, and further efforts are needed in defining its pathogenesis and developing means for its early detection, accurate diagnosis, prevention, and treatment.

\section{Supplementary Material}

Refer to Web version on PubMed Central for supplementary material.

\section{Acknowledgments}

DILIN is supported by the National Institute of Diabetes and Digestive and Kidney Diseases under the following cooperative agreements: 1U01DK065201, 1U01DK065193, 1U01DK065184, 1U01DK065211, 1U01DK065238, and 1U01DK065176.

The authors disclose the following: N.C. served as a paid consultant in the preceding 12 months to Takeda Pharmaceuticals, AtheroGenics, Advanced Life Sciences, Kari Bio, Metabasis, Pfizer, and Eli Lilly. He received research grant support from Debiovision and Sanofi-Aventis. On his behalf, his institution has initiated contract negotiations with Gilead Sciences and Pfizer for conducting clinical trials unrelated to drug-induced liver injury. He served as an expert witness for a product liability litigation involving suspected drug-induced liver injury. 


\section{Abbreviations used in this paper}

$\begin{array}{ll}\text { DILI } & \text { drug-induced liver injury } \\ \text { DILIN } & \text { Drug-Induced Liver Injury Network } \\ \text { INR } & \text { international normalized ratio } \\ \text { RUCAM } & \text { Roussel Uclaf Causality Assessment Method } \\ \text { ULN } & \text { upper limit of normal }\end{array}$

\section{References}

1. Watkins PB, Seeff LB. Drug-induced liver injury: summary of a single topic clinical research conference. Hepatology. 2006; 43:618-631. [PubMed: 16496329]

2. Navarro VJ, Senior JR. Drug-related hepatotoxicity. N Engl J Med. 2006; 35:731-739. [PubMed: 16481640]

3. Abboud G, Kaplowitz N. Drug-induced liver injury. Drug Saf. 2007; 30:277-294. [PubMed: 17408305]

4. Ostapowicz G, Fontana RJ, Schiødt FV, et al. U.S. Acute Liver Failure Study Group. Results of a prospective study of acute liver failure at 17 tertiary care centers in the United States. Ann Intern Med. 2002; 137:947-954. [PubMed: 12484709]

5. Uetrecht J. Idiosyncratic drug reactions: current understanding. Annu Rev Pharmacol Toxicol. 2007; 47:513-539. [PubMed: 16879083]

6. Wilkes RA, Lin DW, Roden DM, et al. Identifying genetic risk factors for serious adverse drug reactions: current progress and challenges. Nat Rev Drug Discov. 2007; 6:904-916. [PubMed: 17971785]

7. Lammert C, Einarsson S, Saha C, et al. Relationship between daily dose of oral medications and idiosyncratic drug-induced liver injury: search for signals. Hepatology. 2008; 47:2003-2007. [PubMed: 18454504]

8. Senior JR. What is idiosyncratic hepatotoxicity? What is not? Hepatology. 2008; 47:1813-1815. [PubMed: 18508312]

9. Seeff LB. Herbal hepatotoxicity. Clin Liver Dis. 2007; 11:577-596. [PubMed: 17723921]

10. Strader, DB.; Seeff, LB. Hepatotoxicity of herbal preparation. Chapter 37. In: Boyer, TD.; Wright, TL.; Manns, MP., editors. Zakim and Boyer's hepatology, a textbook of liver disease. 5th ed. Philadelphia, PA: Saunders-Elsevier; 2006. p. 551-560.

11. Elinav E, Pinsker G, Safadi R, et al. Association between consumption of Herbalife ${ }^{\circledR}$ nutritional supplements and acute hepatotoxicity. J Hepatol. 2007; 47:514-520. [PubMed: 17692424]

12. Schoepfer AM, Engel A, Fattinger K, et al. Herbal does not mean innocuous: ten cases of severe hepatotoxicity associated with dietary supplements from Herbalife ${ }^{\circledR}$ products. J Hepatol. 2007; 47:521-526. [PubMed: 17692989]

13. Sarma DN, Barrett ML, Chavez ML, et al. Safety of green tea extracts: a systematic review by the US Pharmacopeia. Drug Saf. 2008; 31:469-484. [PubMed: 18484782]

14. Bonkovsky HL. Hepatotoxicity associated with supplements containing Chinese Green Tea (Camellia sinensis) (letter). Ann Intern Med. 2006; 144:68-70. [PubMed: 16389263]

15. Javaid A, Bonkovsky HL. Hepatotoxicity due to extracts of Chinese green tea (Camellia sinensis): a growing concern (letter). J Hepatol. 2006; 45:334-336. [PubMed: 16793166]

16. Gardiner P, Graham R, Legedza AT, et al. Factors associated with herbal therapy use by adults in the United States. Altern Ther Health Med. 2007; 13:22-29. [PubMed: 17405675]

17. Uetrecht J. Idiosyncratic drug reactions: past, present, and future. Chem Res Toxicol. 2008; 21:8492. [PubMed: 18052104]

18. Ingelman-Sundberg M. Pharmacogenomic biomarkers for prediction of severe adverse drug reactions. N Engl J Med. 2008; 358:637-639. [PubMed: 18256400] 
19. Eichelbaum M, Ingelman-Sundberg M, Evans WE. Pharmacogenomics and individualized drug therapy. Annu Rev Med. 2006; 57:119-137. [PubMed: 16409140]

20. Hoofnagle JH. Drug-Induced Liver Injury Network (DILIN). Hepatology. 2004; 40:773. [PubMed: 15382161]

21. Fontana RJ, Watkins PB, Bonkovsky HL, et al. Rationale, design and conduct of the Drug-Induced Liver Injury Network (DILIN) prospective study. Drug Saf. (in press).

22. Rockey DC, Seeff LB, Freston JW, et al. Comparison between expert opinion and RUCAM for assignment of causality in drug induced liver injury (abstr M1777). Gastroenterology. 2007; 132(suppl 2):A-773.

23. Benichou C. Criteria of drug induced liver disorders: report of an international consensus meeting. J Hepatol. 1990; 11:272-276. [PubMed: 2254635]

24. Danan G, Benichou C. Causality assessment of adverse reactions to drugs - I A novel method based on the conclusions of international consensus meeting. J Clin Epidemiol. 1993; 46:13231330. [PubMed: 8229110]

25. Benichou C, Danan G, Flahault A. Causality assessment of adverse reactions to drugs-II An original model for validation of drug causality assessment methods: case reports with positive rechallenge. J Clin Epidemiol. 1993; 46:1331-1336. [PubMed: 8229111]

26. Andrade RJ, Lucena MI, Fernandez MC, et al. Drug induced liver injury: an analysis of 461 incidences submitted to the Spanish registry over a 10-year period. Gastroenterology. 2005; 129:512-521. [PubMed: 16083708]

27. De Valle MB, Klinteberg VA, Alem N, et al. Drug induced liver injury in a Swedish University Hospital out-patient hepatology clinic. Aliment Pharmacol Ther. 2006; 24:1187-1195. [PubMed: 17014577]

28. Pillans PI. Drug associated hepatic reactions in New Zealand: 21 years experience. N Z Med J. 1996; 109:315-319. [PubMed: 8816722]

29. Björnsson E, Olsson R. Outcome and prognostic markers in severe drug-induced liver disease. Hepatology. 2005; 42:481-489. [PubMed: 16025496]

30. Ganey PE, Luyendyk JP, Maddox JF, et al. Adverse hepatic drug reactions: inflammatory episodes as consequence and contributor. Chem Biol Interact. 2004; 150:35-51. [PubMed: 15522260]

31. Dalton HR, Fellows HJ, Stableforth W, et al. The role of hepatitis E virus testing in drug-induced liver injury. Aliment Pharmacol Ther. 2007; 26:1429-1435. [PubMed: 17850420]

32. El-Serag HB, Everhart JE. Diabetes increases the risk of acute hepatic failure. Gastroenterology. 2002; 122:1822-1828. [PubMed: 12055590]

33. Wang T, Shankar K, Ronis MJ, et al. Mechanisms and outcomes of drug-and toxicant-induced liver toxicity in diabetes. Clin Rev Toxicol. 2007; 37:413-459.

34. Mehendale, HM. Role of tissue repair in liver injury. In: Kaplowitz, N.; Delve, L., editors. Drug induced liver injury. 2nd ed.. Philadelphia, PA: Informa Health Care; 2007.

35. Björnsson E, Kalaitzakis E, Olsson R. The impact of eosinophilia and hepatic necrosis on prognosis in patients with drug-induced liver injury. Aliment Pharmcol Ther. 2007; 25:1411-1421.

36. Ohmori S, Shiraki K, Inoue H, et al. Clinical characteristics and prognostic indicators of druginduced fulminant hepatic failure. Hepatogastroenterology. 2003; 50:1531-1534. [PubMed: 14571779]

37. Zimmerman, HJ. Hepatotoxicity: the adverse effects of drugs and other chemicals on the liver. 2nd ed. Philadelphia, PA: Lippincott, Williams and Wilkins; 1999. Drug-induced liver disease. Chapter 16; p. 427-456.

38. Björnsson E. Drug-induced liver injury: Hy’s rule revisited. Clin Pharmacol Ther. 2006; 79:521528. [PubMed: 16765139]

39. Sgro C, Clinard F, Ouazir K, et al. Incidence of drug-induced hepatic injuries: a French populationbased study. Hepatology. 2002; 36:451-455. [PubMed: 12143055]

\section{Appendix}

Full Listing of the DILIN Members 


\section{Clinical Centers}

Indiana University

Naga Chalasani, MD (PI), Raj Vuppalanchi, MD (co-I), Jean Molleston, MD (co-I), Lawrence Lumeng, MD (co-I), Audrey Corne (research coordinator), Angie Plummer (research coordinator)

University of Connecticut

Herbert Bonkovsky, MD (PI), Petr Protiva, MD (co-I), James Freston, MD, PhD (co-I), Robert Rosson, MD (co-I), Robert A. Levine, MD (Satellite site investigator), Benedict Maliakkal, MD (Satellite site investigator), Paul Appleton, MD (Research coordinator), Mariola Smialek, RN (research coordinator)

University of Michigan

Robert J. Fontana, MD (PI), Hari Conjeevaram, MD (co-I), Stuart Gordon, MD (Satellite site investigator), Suzanne Welch (research coordinator), Jessica Worley (research coordinator); Jordan Kridler (research coordinator).

University of North Carolina

Paul Watkins, MD (PI), Paul Hayashi, MD (co-I), Mark Russo, MD (co-I), Late Harry Guess, MD, PhD (co-investigator), Kimberly Beaver, MD (Satellite site investigator), Alastair Smith, MD (Satellite site investigator), James Lewis, MD (Satellite site investigator), Susan Pusek (Research coordinator)

University of California, San Francisco

Tim Davern, MD (PI), Maurizo Bonacini, MD (co-I), Kristine Partovi (research coordinator)

\section{Data Coordinating Center}

Duke Clinical Research Institute

James Rochon, MD (PI), John McHutchison, MD (co-I), Don Rockey, MD (co-I), Mary Maggio (project manager), Hongqiu Yang, $\mathrm{PhD}$ (biostatistician)

\section{NIDDK Scientists}

Jose Serrano, MD (Project officer), Leonard Seeff, MD, Jay Hoofnagle, MD

*Mark Avigan, MD and John Senior, MD, employees of the U.S. Food and Drug Administration have participated in selected aspects of the DILIN activities. 


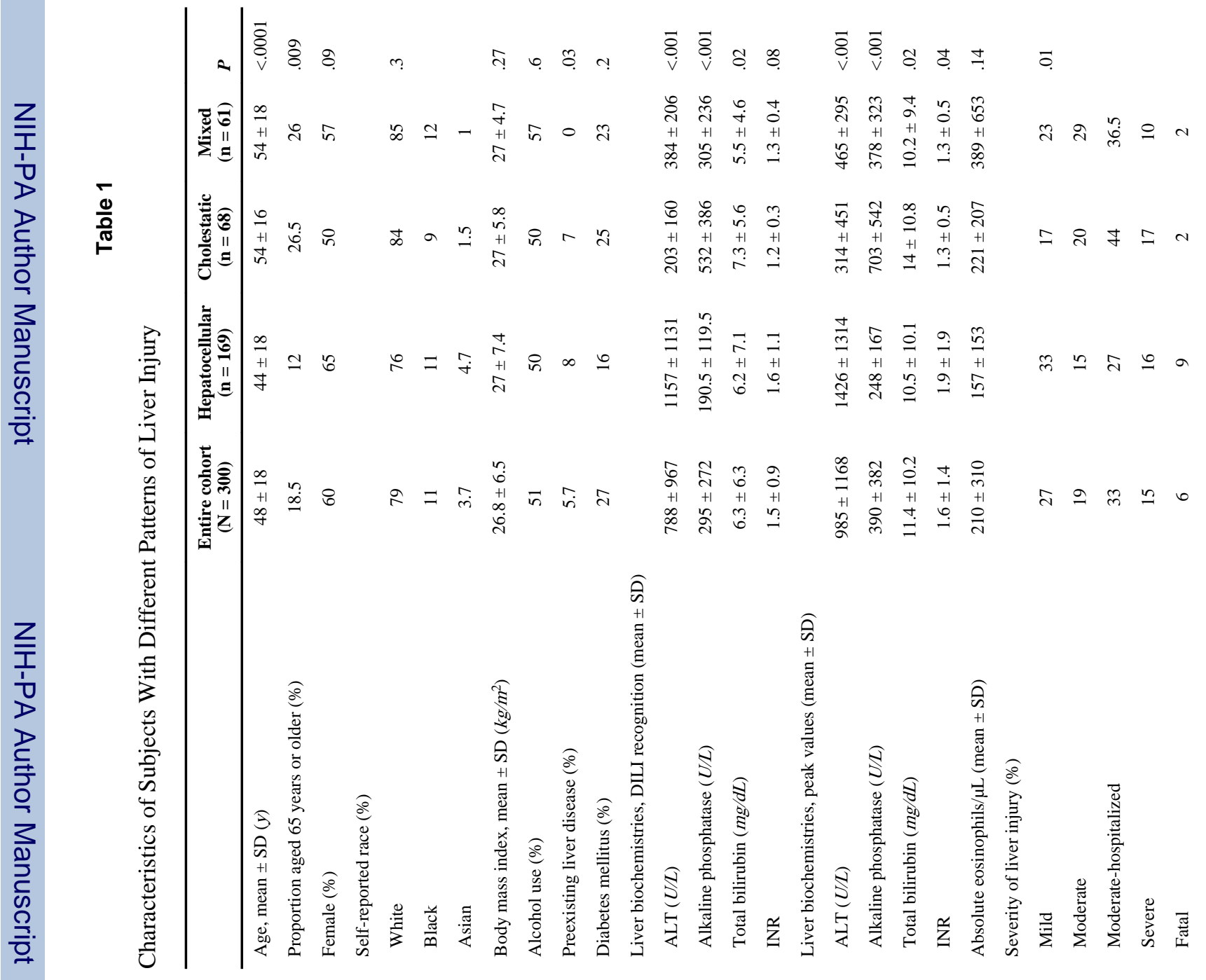




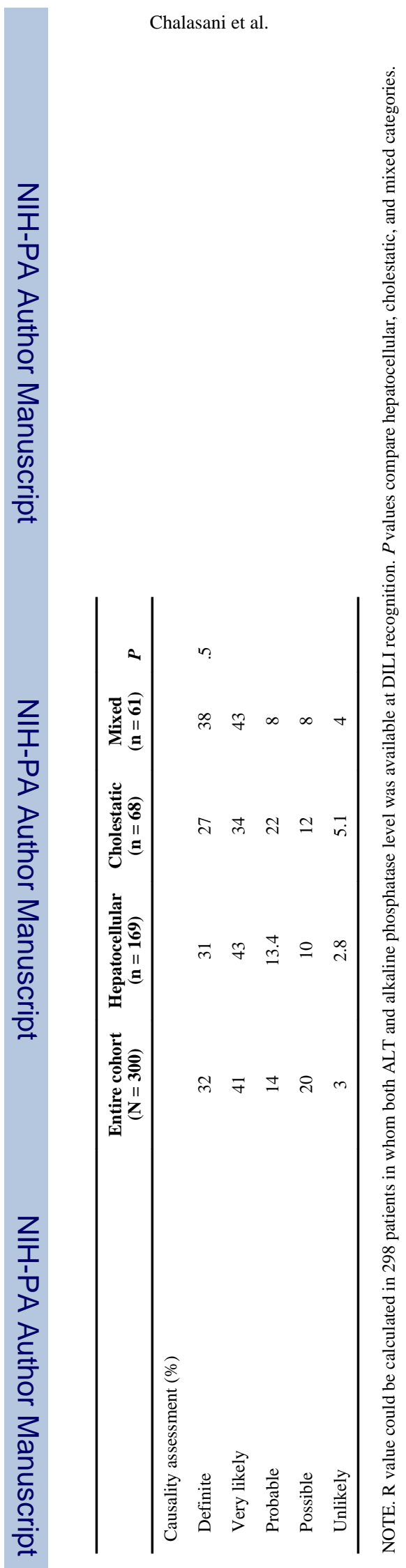

Page 14

要


Table 2

Clinical Characteristics of DILI Subjects Categorized by Liver Injury Severity

\begin{tabular}{|c|c|c|c|}
\hline & $\begin{array}{c}\text { Mild/moderate DILI } \\
\quad(n=195)\end{array}$ & $\begin{array}{l}\text { Severe DILI } \\
\quad(\mathbf{n}=\mathbf{5 1})\end{array}$ & $P$ \\
\hline Age, mean $\pm \mathrm{SD}(y)$ & $50 \pm 18$ & $46 \pm 16.3$ & .15 \\
\hline Proportion aged $\prec 65$ years $(\%)$ & 23 & 11 & .06 \\
\hline Female $(\%)$ & 57 & 59 & .80 \\
\hline \multicolumn{4}{|l|}{ Self-reported race $(\%)$} \\
\hline White & 81 & 67 & .10 \\
\hline Black & 11 & 18 & \\
\hline Asian & 2 & 8 & \\
\hline Body mass index, mean $\pm \mathrm{SD}\left(\mathrm{kg} / \mathrm{m}^{2}\right)$ & $26.4 \pm 5.9$ & $27.2 \pm 7.8$ & .90 \\
\hline Alcohol use (\%) & 57 & 32 & .001 \\
\hline Preexisting liver disease (\%) & 6 & 9 & .50 \\
\hline Diabetes (\%) & 25 & 37 & .07 \\
\hline Days between exposure and DILI recognition, median ( 25 th, 75 th percentiles) & $35.5(19,89)$ & $65.5(33,263)$ & .006 \\
\hline \multicolumn{4}{|l|}{ Implicated agent(s) $(\%)$} \\
\hline Single prescription agent & 74 & 80 & .40 \\
\hline Single or multiple dietary supplements & 7.2 & 2 & \\
\hline Multiple prescription or prescription plus dietary supplements & 19 & 18 & \\
\hline \multicolumn{4}{|l|}{ Liver biochemistries, values at DILI onset (mean $\pm \mathrm{SD}$ ) } \\
\hline $\operatorname{ALT}(U / L)$ & $610 \pm 685$ & $1218 \pm 1559$ & $<.001$ \\
\hline Alkaline phosphatase $(U / L)$ & $309 \pm 284$ & $283 \pm 247$ & .60 \\
\hline Bilirubin $(m g / d L)$ & $5.2 \pm 5.1$ & $9.1 \pm 8.4$ & $<.001$ \\
\hline INR & $1.2 \pm 0.3$ & $2.3 \pm 1.6$ & $<.001$ \\
\hline \multicolumn{4}{|l|}{ Liver biochemistries, peak values (mean $\pm \mathrm{SD}$ ) } \\
\hline $\operatorname{ALT}(U / L)$ & $733 \pm 726$ & $1513 \pm 1734$ & $<.001$ \\
\hline Alkaline phosphatase $(U / L)$ & $388 \pm 399$ & $401 \pm 354$ & .001 \\
\hline Bilirubin $(m g / d L)$ & $8.9 \pm 9.4$ & $18.4 \pm 10.2$ & .50 \\
\hline INR & $1.2 \pm 0.3$ & $2.9 \pm 2.5$ & $<.001$ \\
\hline Absolute eosinophil count $/ \mu \mathrm{L}$ (mean $\pm \mathrm{SD}$ ) & $195 \pm 344$ & $197 \pm 293$ & .70 \\
\hline \multicolumn{4}{|l|}{ Pattern of liver injury $(\%)$} \\
\hline Hepatocellular & 50 & 69 & .06 \\
\hline Cholestatic & 27 & 19 & \\
\hline Mixed & 23 & 12.5 & \\
\hline \multicolumn{4}{|l|}{ Causality score } \\
\hline Definite & 32 & 25.5 & .17 \\
\hline Highly likely & 41.5 & 35 & \\
\hline Probable & 13 & 22 & \\
\hline Possible & 10 & 10 & \\
\hline Unlikely & 2.6 & 8 & \\
\hline Chronic DILI (\%) & 17 & 12 & 1.0 \\
\hline Liver-related mortality (\%) & 0 & 23 & $<.001$ \\
\hline
\end{tabular}




\begin{tabular}{lccc}
\hline & $\begin{array}{c}\text { Mild/moderate DILI } \\
(\mathbf{n}=\mathbf{1 9 5})\end{array}$ & $\begin{array}{c}\text { Severe DILI } \\
(\mathbf{n}=\mathbf{5 1})\end{array}$ & $\boldsymbol{P}$ \\
\hline Liver transplantation $(\%)$ & 0 & 2.9 & .17 \\
\hline
\end{tabular}

Mild DILI: elevated ALT and/or alkaline phosphatase level but serum total bilirubin level $<2.5 \mathrm{mg} / \mathrm{dL}$ and INR $<1.5$; moderate DILI: elevated ALT and/or alkaline phosphatase levels and serum total bilirubin $\geq 2.5 \mathrm{mg} / \mathrm{dL}$ or INR $\geq 1.5$; moderate-severe DILI: elevated ALT, alkaline phosphatase, bilirubin, and/or INR levels and patient is hospitalized for DILI or if ongoing hospitalization is prolonged; severe DILI: elevated ALT and/or alkaline phosphatase level and serum bilirubin level $\geq 2.5 \mathrm{mg} / \mathrm{dL}$ and hepatic failure (INR $\geq 1.5$, ascites or encephalopathy); fatal/ transplant: patient dies or undergoes transplantation because of DILI event. Advanced DILI consisted of severe and fatal/transplant cases, whereas nonadvanced DILI consisted of 3 other categories. 


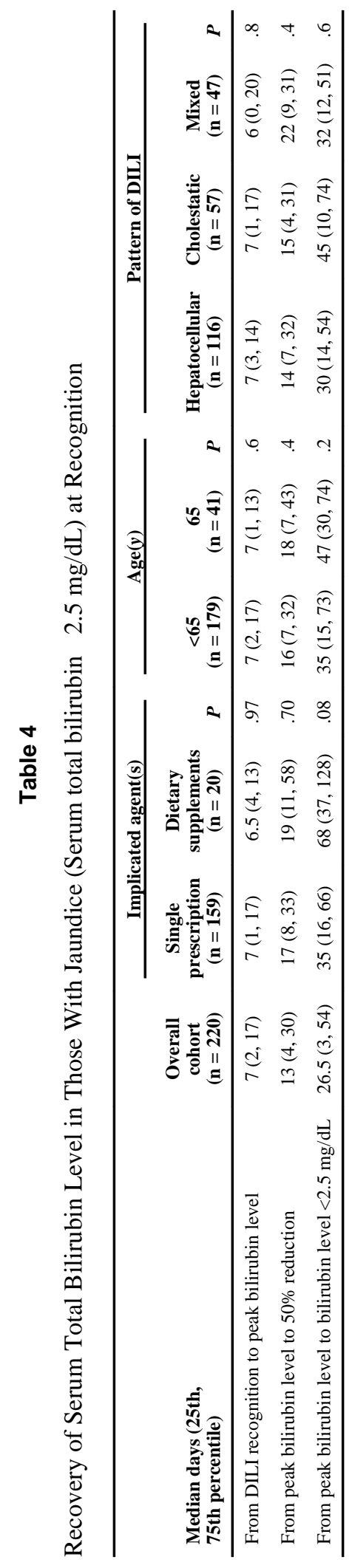




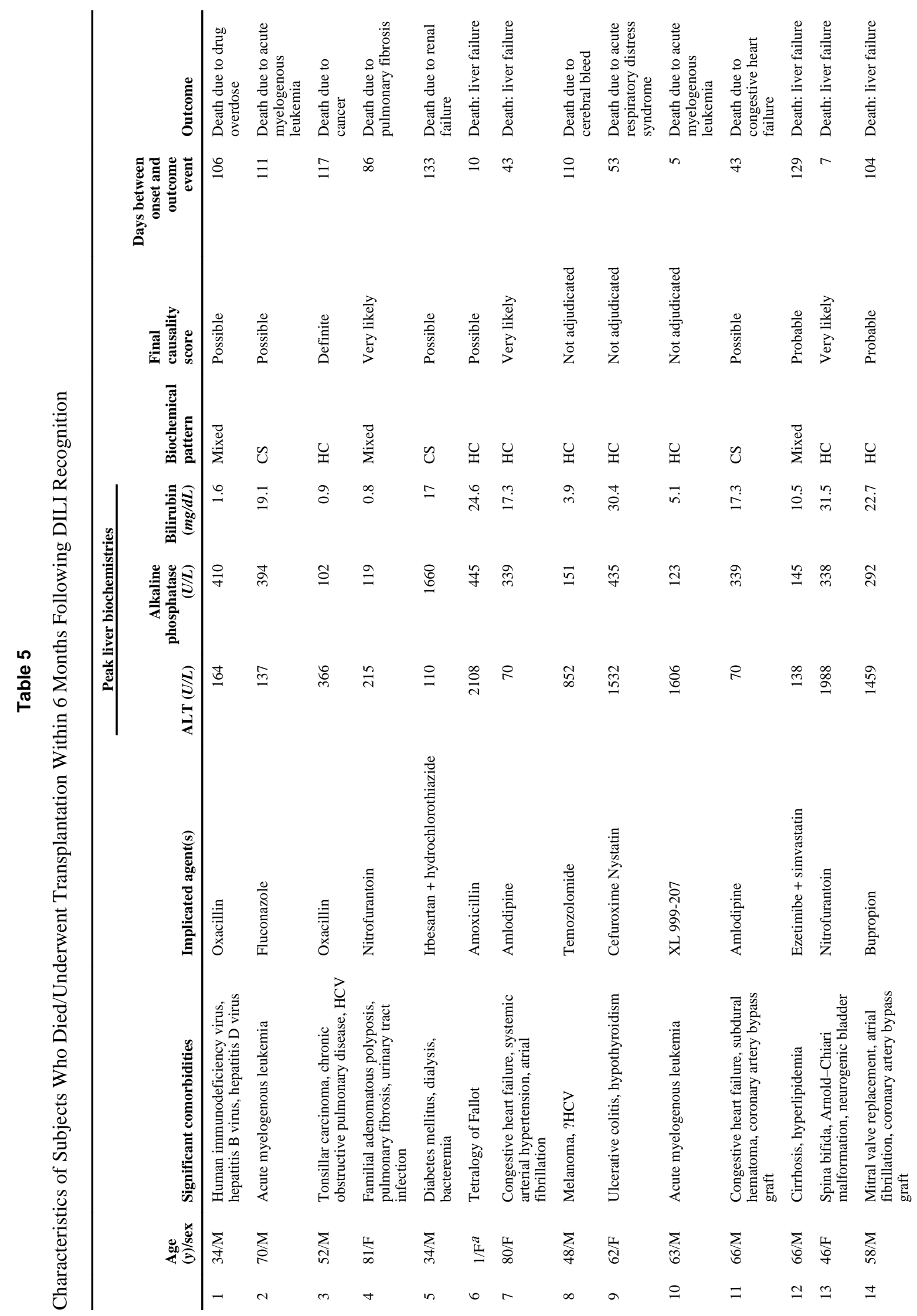

Gastroenterology. Author manuscript; available in PMC 2013 May 15. 


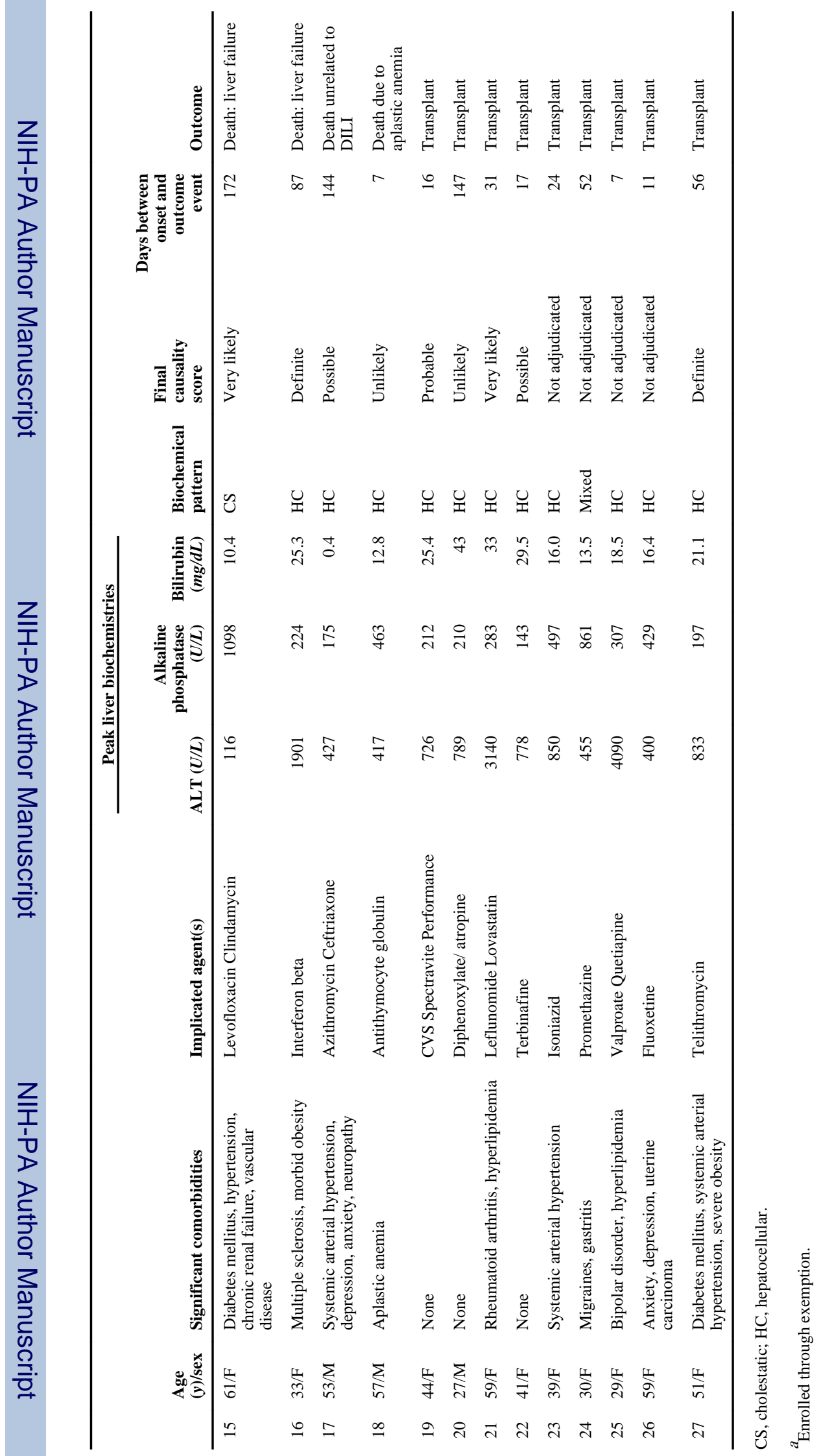


Table 6

Mortality Rates and Biochemical Injury Pattern Reported in Recent Reports

\begin{tabular}{lccl}
\hline Reference & $\begin{array}{c}\text { Hepatocellular } \\
(\boldsymbol{\%})\end{array}$ & $\begin{array}{c}\text { Cholestatic } \\
(\boldsymbol{\%})\end{array}$ & $\begin{array}{l}\text { Mixed } \\
(\boldsymbol{\%})\end{array}$ \\
\hline Björnsson and Olsson $^{29}$ & 12.7 & 7.8 & 2.4 \\
${\text { Andrade et } \mathrm{al}^{26}}^{\text {Chalasani et al (current report) }}$ & 7 & 5 & 2 \\
\hline
\end{tabular}

